\title{
A Study of Martian Topography by Analytic Photogrammetry ${ }^{1}$
}

\author{
KarL R. BLasius \\ Division of Geological Sciences, California Institute of Technology \\ Pasadena, California 91109
}

\begin{abstract}
Many overlapping pictures, potential data for the construction of topographic maps, were obtained by the television cameras on Mariner 9. An analysis of the sources of error in photogrammetric determinations of relief from these pictures singles out photo resolution as the primary limiting factor. Topographic maps of several Martian surface features, derived by an original analytic scheme, are presented. The observed errors in relief determinations using this technique are in good agreement with the independent error analysis.
\end{abstract}

When the television experiment for the Mariner Mars 1971 Project was planned, the stereometric potential of the television cameras was ignored in favor of scientific objectives that required only single pictures or multiple coverage without a requirement of different viewing perspectives [Masursky et al., 1970].

However, as the mission developed, with one spacecraft instead of two and with a severe dust storm obscuring surface features, a reevaluation was required, and much day-to-day improvisation was necessary. As a result, early in the mission we obtained significant multiple photographic coverage of the few surface features that were relatively clear of intervening dust. In the equatorial regions of Mars four of these features proved to be great volcanic shields with local relief of the order of tens of kilometers. This discovery, coupled with the availability of many overlapping pictures, made photogrammetry from Mariner 9 pictures an exciting possibility.

While the mission was still in the planning stage, it was realized that the potential for photogrammetry might exceed original expectations, so an analytic routine was prepared to test the quality of any potential stereo pairs for determining relative relief of point features seen in two pictures taken from sufficiently different camera stations (see the appendix). This

${ }^{1}$ Contribution 2250, Division of Geological Sciences, California Institute of Technology, Pasadena, California.

Copyright $\subset 1973$ by the American Geophysical Union. technique now has yielded several stereo models of surface features of great geologic interest and has demonstrated the potential of the Mariner 9 pictures for construction of contoured topographic maps through the use of stereo plotters. This latter work is in progress, and its first results are now being published [Wu et al., 1973]. This paper discusses the photogrammetric potential of Mariner 9 data and the analytically determined topography of certain Martian landforms.

\section{Precision of Relief Measurement}

The photographic phenomenon that allows height measurement from photography is termed relief displacement. Referring to Figure 1, in which an object $\mathbf{H}$ of height $H$ imaged by a camera of focal length $f$ is represented, we see that the image of the top of $\mathbf{H}$ is formed at a point farther from the nadir image $N^{\prime}$ than is the image at the foot of $\mathbf{H}$. The distance $H^{\prime}$ between these two image points is the relief displacement of the top of $\mathbf{H}$ due to its elevation. If $\mathbf{H}$ is imaged within a small angle of the camera axis (a very good approximation for the Mariner cameras, whose angular fields are only $1.1^{\circ} \times 1.4^{\circ}$ and $11^{\circ} \times$ $14^{\circ}$ ), these quantities are approximately related by the equation

$$
H=H^{\prime} / \sin (V A R) S R / f
$$

where $S R$ is the slant range of the camera from the feature and $V A R$ is the viewing angle at the feature measured from the local vertical. If we take partial derivatives of (1), we obtain an equation for small changes, $\Delta H$, in any 




Fig. 1. Basic elements of the geometry of an oblique planetary scale picture in the principal plane (the plane containing the nadir and the camera axis).

relief determination in terms of small changes in the other quantities:

$$
\Delta H=C_{0} \Delta H^{\prime}+C_{1} \Delta f+C_{2} \Delta S R
$$

$$
+C_{3} \Delta V A R
$$

where

$$
\begin{aligned}
C_{0} & \equiv S R /[f \cdot \sin (V A R)] \\
C_{1} & \equiv-H^{\prime} / \sin (V A R) S R / f^{2} \\
C_{2} & \equiv H^{\prime} / \sin (V A R) 1 / f \\
C_{3} & \equiv\left[-H^{\prime} \cdot \cos (V A R)\right] / \sin ^{2}(V A R) S R / f
\end{aligned}
$$

The quantities whose variations are shown in (2) to contribute to $\Delta H$ fall into two distinct categories according to their effect on a stereo model. Many determinations of $H^{\prime}$ go into the construction of a single stereo model, and so random errors in the measurement process may appear as inconsistencies in the derived topography (see the appendix). Errors in $f, S R$, or $V A R$, on the other hand, create an overall model scaling error. We shall find that predicted random errors in $H^{\prime}$ account well for the inconsistencies observed in the stereo models and should dominate the effects of errors in $f, S R$, and $V A R$ in wide angle Mariner 9 photography.

For a sample error analysis consider the simple case where one picture of a pair was taken from an oblique perspective (the case of Figure 1) and the other was taken from a vertical perspective $(V A R=0)$. In Table 1 typical values of the parameters of (1) are given for the members of such a Mariner 9 picture pair. The uncertainties in some of the quantities on the right-hand side of (1) can be estimated from work performed by JPL scientists. Preflight calibration data [Snyder, 1971] gives 1- $\sigma$ uncertainties in the focal lengths as listed in Table 1.

In creating a self-consistent stereo model (see the appendix) the slant range $S R$ may have to be changed from the nominal value for one of the pictures, and so the unchanged $S R$ of the other picture sets the scale for the model. The basic quantities involved in calculating $S R$ are the radius of the planet $R$, the range to the center of the planet $R M A G$, and the tilt angle of the camera axis away from the direction to the center of the planet. For the small tilt angles of our examples (less than $20^{\circ}$ for the oblique pictures) the probable error in $S R, \triangle S R$, is due to $\Delta R$ and $\triangle R M A G$. Both preflight and post-flight analyses agree that there is a $5-\mathrm{km}(1-\sigma)$ uncertainty in calculated values of $R M A G$ at periapsis (E. J. Christensen, personal communication, 1972). This error scales approximately as a constant percentage of $R M A G$. For the largest values of $R M A G$ that affect our models, we find $\triangle R M A G=8 \mathrm{~km}$.

Estimating Mars' radius at any location is a very subjective matter, since preliminary and scattered data must be combined from several sources, such as Mariner 9 occultation and spectrometer experiments and earth-based radar data. On the basis of such an informal procedure, we estimate a probable error of $5 \mathrm{~km}$ in the assumed values of Mars' radius. In combination with the value of $\triangle R M A G$ given above, we find $\Delta S R \simeq 10 \mathrm{~km}$.

A Jet Propulsion Laboratory (JPL) study of the precision of the spacecraft pointing angles from the positions of ground features in re-

\begin{tabular}{|c|c|c|c|c|c|}
\hline Camera & $\begin{array}{l}S R \\
\mathrm{~km}\end{array}$ & $f( \pm 1 \sigma)$, & $\begin{array}{l}V A R \\
\text { deg }\end{array}$ & $\begin{array}{l}H, \\
\text { kin }\end{array}$ & $\begin{array}{l}H^{\prime} \text { Derived } \\
\text { by Using } \\
\text { (1), } \\
\text { mm }\end{array}$ \\
\hline $\begin{array}{l}\text { Wide angle } \\
\text { Narrow } \\
\text { angle }\end{array}$ & $\begin{array}{l}2 \times 10^{3} \\
2 \times 10^{3}\end{array}$ & $\begin{array}{r}52.267( \pm 0.006) \\
500.636( \pm 0.036)\end{array}$ & $\begin{array}{l}30 \\
30\end{array}$ & $\begin{array}{l}5 \\
5\end{array}$ & $\begin{array}{l}6.25 \times 10^{-2} \\
6.25 \times 10^{-1}\end{array}$ \\
\hline
\end{tabular}
peated photography (S. Mohan, personal communication, 1973) concluded that they were generally accurate to better than $0.1^{\circ}$. This

TABLE 1. Typical Values of Photogrammetric Parameters Associated with Mariner 9 Pictures of Martian Surface 
translates into a $\triangle V A R$ of about $0.1^{\circ}$ for each photo of our sample stereo model, since they were not taken from extreme altitudes or extremely oblique perspectives. The total $\triangle V A R$ for a model, if the errors are assumed to be uncorrelated, is then approximately $0.15^{\circ}$.

The uncertainty in $H^{\prime}$ is related to the accuracy with which the distance between point features can be determined in the camera focal plane. Two factors are involved here: the spatial resolution of the cameras and the geometric fidelity of the processed pictures. There is no single figure for spatial resolution, since this characteristic is a function of the contrast of the target scene. A discussion of this problem in connection with photography from spacecraft is contained in a recent paper [Masursky et al., 1970]. For this error analysis I use a spatial resolution of $0.027 \mathrm{~mm}$ corresponding to a high-contrast target such as a steep-walled crater illuminated by a sun low in the sky. The point features to be located are the centers of such resolved features. This is easily done to an accuracy of $0.007 \mathrm{~mm}$. (The point features chosen to construct the stereo models presented later in this paper closely approximate this ideal.) The derivation of one elevation requires the location of four image points, the same pair of features in two pictures. Then $H^{\prime}$ is derived from the difference in the image distances between that pair of features.

The geometric fidelity of the pictures is a complicated problem that involves the preflight measurement of electronic and optical disţortions created by the cameras and their removal from returned pictures. A detailed discussion of this procedure is now in preparation (J. Kreznar, unpublished manuscript, 1973). In part of the calibration routine a square grid of lines was used. Accurate determinations of the coordinates of about 400 intersections on that grid can be compared with their coordinates on a geometrically corrected image of the grid. (It is believed that there are several uncalibrated sources of systematic, but small, errors in the image coordinates.) For both Mariner 9 cameras the rms separation of the actual intersection positions from their images is less than $0.004 \mathrm{~mm}$ (J. Kreznar, personal communication, 1972). If we assume that this figure can be applied to the Mariner 9 pictures generally, we can estimate the probable error for a single point feature position determination $\Delta P^{\prime}$ by combining the effects of picture resolution and geometric distortion:

$$
\begin{array}{r}
\Delta P^{\prime} \approx\left[(0.007)^{2}+(0.004)^{2}\right]^{1 / 2} \\
=0.008 \mathrm{~mm}
\end{array}
$$

Picture resolution is the dominant source of error here. The uncertainty in $H^{\prime}$, due to four such determinations, is estimated as

$$
\Delta H^{\prime} \approx\left[4\left(\Delta P^{\prime}\right)^{2}\right]^{1 / 2}=0.016 \mathrm{~mm}
$$

With the foregoing estimates of the parameters in (1) and their probable errors, the terms of the right-hand side of (2) can be evaluated to check their relative importance. Table 2 lists their calculated values. It is clear that the first term is by far the most important for wide angle photography; errors due to $\Delta H^{\prime}$, primarily owing to photoresolution, should dominate over the three sources of error in overall model scale. Elevation differences in this model would have to be stated with \pm 1300 meters independent of their magnitude.

For the narrow angle photography, $\triangle V A R$ should result in an uncertainty in elevation differences of about $4.5 \%$ (225 meters in 5000 meters) in addition to \pm 130 meters due to $\Delta H^{\prime}$.

A shortened form of (2), including only the term due to $\Delta H^{\prime}$, can be used to predict the scatter of relief determinations $\Delta H$ in our stereo models:

$$
\Delta H \approx \frac{S R}{f \cdot \sin (V A R)} 1.6 \times 10^{-5} \text { meters }
$$

A slight elaboration of the meaning of (6) must

\begin{tabular}{|c|c|c|}
\hline Coefficient & Probable Error & $\begin{array}{l}\text { Contribution to } \\
\text { Probable Error in } \\
H=5000 \text { meters } \\
\text { meters }\end{array}$ \\
\hline & Wide Angle Camera & \\
\hline$c_{0}=8 \times 10^{7}$ & $\Delta_{H}{ }^{\prime}=0.016 \mathrm{~mm}$ & $C_{0} \Delta_{H^{\prime}}=1280$ \\
\hline$c_{1}=10^{5}$ & $\hbar_{f}(1 \quad \sigma)=0.006 \mathrm{~mm}$ & $C_{1} \vec{\Delta}_{f}=0.6$ \\
\hline$C_{2}=2.5 \times 10^{-3}$ & $\Delta_{S R}=10 \mathrm{~km}$ & $c_{2} \overrightarrow{\Delta S R}=25$ \\
\hline \multirow{2}{*}{$C_{3}=\underset{\text { meters }}{8.6 \times 10^{3}}$} & $\nabla_{V A R}=0.15^{\circ}$ & $C_{3} \overleftarrow{\Delta} V A R=225$ \\
\hline & Narras Angle Camera & \\
\hline$c_{0}=8 \times 10^{6}$ & $\nabla_{H^{\prime}}=0.016 \mathrm{~mm}$ & $c_{0} \vec{\Delta}_{H^{\prime}}=128$ \\
\hline$c_{1}=10^{4}$ & $\vec{\Delta} f(1 \quad \sigma)=0.036 \mathrm{mul}$ & $c_{1} \Delta_{f}=0.36$ \\
\hline$c_{2}=2.5 \times 10^{-3}$ & $\hbar S R=10 \mathrm{~km}$ & $c_{2} \pi_{S R}=25$ \\
\hline$C_{3}=\underset{\substack{8.6 \\
\text { meters }}}{ } \times 10^{3}$ & $\nabla_{V A R}=0.15^{\circ}$ & $C_{3} \overrightarrow{\Delta V A R}=225$ \\
\hline
\end{tabular}
be added. The $\Delta H$ calculated by (6) applies to a

TABLE 2. Calculated Values from Evaluation of Terms of Right-Hand Side of (2) 
stereo picture pair consisting of one picture taken from a vertical perspective and a second from an oblique perspective. If, instead, we have the more general case of two oblique pictures with the camera stations lying in a vertical plane at the target on opposite sides of the target, the cumulative relief displacement in the two pictures (subscripts 1 and 2) is

$$
H_{T}{ }^{\prime} \equiv H_{1}{ }^{\prime}+H_{2}{ }^{\prime}
$$

where, from (1),

$$
\begin{aligned}
& H_{1}{ }^{\prime}=H \cdot \sin \left(V A R_{1}\right) \cdot\left(f_{1} / S R_{1}\right) \\
& H_{2}{ }^{\prime}=H \cdot \sin \left(V A R_{2}\right) \cdot\left(f_{2} / S R_{2}\right)
\end{aligned}
$$

Thus $H$ and $\mathbf{\Delta} H$ are given by more general forms of (1) and (6):

$$
H=\frac{H_{T^{\prime}}{ }^{\prime}}{\left[\frac{f_{1}}{S R_{1}} \cdot \sin \left(V A R_{1}\right)+\frac{f_{2}}{S R_{2}} \cdot \sin \left(V A R_{2}\right)\right]}
$$

$$
\begin{array}{r}
\Delta H \\
\left.=\frac{1.6 \times 10^{-5} \text { meters }}{S R_{1}} \cdot \sin \left(V A R_{1}\right)+\frac{f_{2}}{S R_{2}} \cdot \sin \left(V A R_{2}\right)\right]
\end{array}
$$

In the most general case the camera stations of a stereo pair do not lie in a plane intersecting the ground target vertically. Then only the components of $V A R_{1}$ and $V A R_{2}$ in a vertical plane at the target contribute to relief determinations. In Figure 2 we represent the ground



Fig. 2. Geometry of two camera stations from which a stereo pair of oblique pictures may be taken. The breakdown of the viewing angle $V A R_{1}$ into perpendicular components is illustrated. Only $\phi_{1}$ contributes to relief determinations. target, the camera stations, and the ground trace of a vertical plane through the target, labeled Great Circle. We now find the components of $V A R_{1}$ and $V A R_{2}$ in this plane. This is most easily done by using a well-known identity for right spherical triangles. In Figure 2 the two perpendicular components of $V A R_{1}$ are designated $\phi_{1}$ (in our vertical plane) and $\omega_{1}$ (opposite angle $W_{1}$ ). Then $\phi_{1}$ is given by

$$
\tan \left(\phi_{1}\right)=\tan \left(V A R_{1}\right) \cos \left(W_{1}\right)
$$

similarly

$$
\tan \left(\phi_{2}\right)=\tan \left(V A R_{2}\right) \cos \left(W_{2}\right)
$$

By using (1), (6), (11a), and (11b), we obtain generalized forms of (1) and (6):

$$
\begin{gathered}
H=\frac{H_{T}{ }^{\prime}}{\left[\frac{f_{1}}{S R_{1}} \cdot \sin (\phi)+\frac{f_{2}}{S R_{2}} \cdot \sin \left(\phi_{2}\right)\right]} \\
\Delta H=\frac{1.6 \times 10^{-5} \text { meters }}{\left[\frac{f_{1}}{S R_{1}} \cdot \sin \left(\phi_{1}\right)+\frac{f_{2}}{S R_{2}} \cdot \sin \left(\phi_{2}\right)\right]}
\end{gathered}
$$

Table 3 lists predicted errors in $H$ for stereo models presented in the next part of this paper. These were calculated by using (13).

\section{Stereo Models}

The topography of a number of interesting Martian landforms has been derived by using our analytic technique of photogrammetry (see the appendix). This report has two purposes, the validation of the technique and a demonstration of the quality of the data for a more thorough analysis. We confine ourselves here to a minimum of geologic interpretation. Such interpretation and speculation is the continuing work of this author and many others both inside and outside the Mariner Mars 1971 Project.

The essentials of our first two stereo models, designated model 1 and model 2, are displayed pictorially in Figure 3, a photomosaic of a large volcanic cone with a central crater. This feature has been named Middle Spot, a reference to its two similar neighbors to the northeast and southwest and its dark dotlike appearance on low-resolution pictures of Mars taken early in the Mariner 9 mission. Centers of circles in Figure 3 mark point features whose elevations have been 
TABLE 3. Stereo Models of Martian Topography Presented in This Paper

\begin{tabular}{|c|c|c|c|c|c|c|c|}
\hline Mode1 & $\begin{array}{l}\text { Photomap } \\
\text { Figure }\end{array}$ & Feature & $\begin{array}{l}\text { Approxinate } \\
\text { Location }\end{array}$ & $\begin{array}{l}\text { Photo } \\
\text { DAS } \\
\text { Time }\end{array}$ & $\begin{array}{c}\text { Camera } \\
\text { Focal } \\
\text { Length, } \\
m m\end{array}$ & $\begin{array}{c}\text { Observed } \\
\text { General Error } \\
\text { in Model } \\
\text { Elevations, } \\
\text { meters }\end{array}$ & $\begin{array}{l}\bar{\Delta}_{H} \text { Predicted } \\
\text { Error Calculated } \\
\text { from (13). } \\
\text { meters }\end{array}$ \\
\hline 1 & 3 & Middle Spot & $113 .{ }^{\circ} \mathrm{W}, 0.5^{\circ} \mathrm{N}$ & $\begin{array}{l}3858340 \\
8585894 \\
4402135\end{array}$ & $\begin{array}{l}500.64 \\
52.267 \\
52.267\end{array}$ & 200 & 300 \\
\hline 2 & 3 & Middle Spot & $113 .{ }^{\circ} \mathrm{W}, 0.5^{\circ} \mathrm{N}$ & $\begin{array}{l}7111128 \\
6029803\end{array}$ & $\begin{array}{r}52.267 \\
500.64\end{array}$ & 400 & 700 \\
\hline 3 & 6 & Residual south polar cap & $356 .{ }^{\circ} \mathrm{W}, 86.3^{\circ} \mathrm{S}$ & $\begin{array}{l}0029803 \\
8331829 \\
5741963\end{array}$ & $\begin{array}{l}500.64 \\
500.64 \\
500.64\end{array}$ & 300 & 120 \\
\hline 4 & 6 & Residual south polar cap & $5 .{ }^{\circ} \mathrm{W}, 86.8^{\circ} \mathrm{S}$ & $\begin{array}{l}7791983 \\
5492378\end{array}$ & $\begin{array}{l}500.64 \\
52.267\end{array}$ & 300 & 130 \\
\hline 5 & 7 & Nix Olympica & $133 .{ }^{\circ} \mathrm{W}, 18 .{ }^{\circ} \mathrm{N}$ & $\begin{array}{r}6823918 \\
10132929\end{array}$ & $\begin{array}{l}52.267 \\
500.64\end{array}$ & 1000 & 1350 \\
\hline 6 & 8 & Intratrough ridge & $87.1^{\circ} \mathrm{W}, 7.3^{\circ} \mathrm{S}$ & 7326763 & 500.64 & 90 & 130 \\
\hline
\end{tabular}

determined analytically. Using four pictures, we have been able to establish two independent stereo models of Middle Spot. Elevations in kilometers are given in parentheses next to the circles as $(1,2)$ derived from model 1 and model 2, respectively. One point has been chosen arbi- trarily as the zero elevation for both models. The photo identification numbers and other information concerning the models are given in Table 3. The column labeled general error in model elevations contains a figure that is half the largest spread of values for the relief between

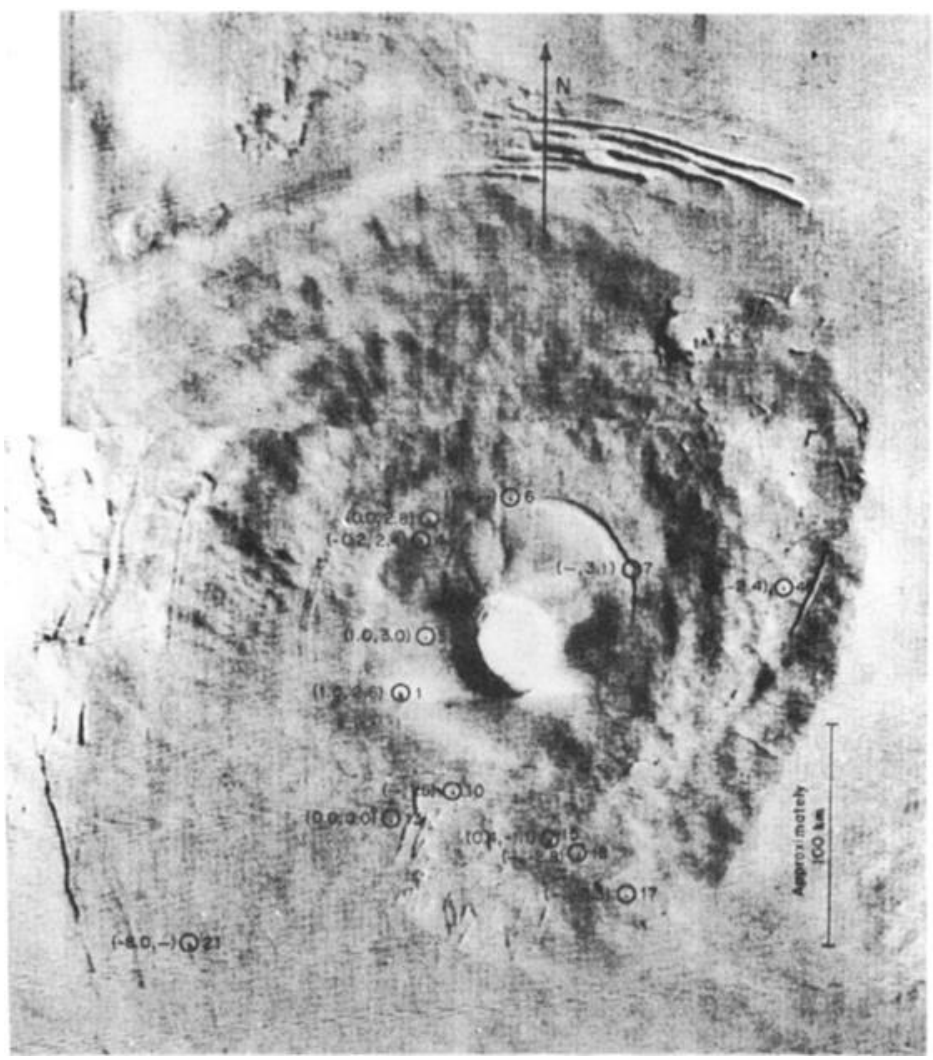

Fig. 3. A mosaic of the Martian volcanic shield Middle Spot. The pictures have been rectified to a vertical perspective. Point elevations in parentheses have been determined by analy tic photogrammetry from models 1 and $2(1,2)$. (MTVS 4184-54, DAS 07111128; MTVS 4184-60, DAS 07111198.) 

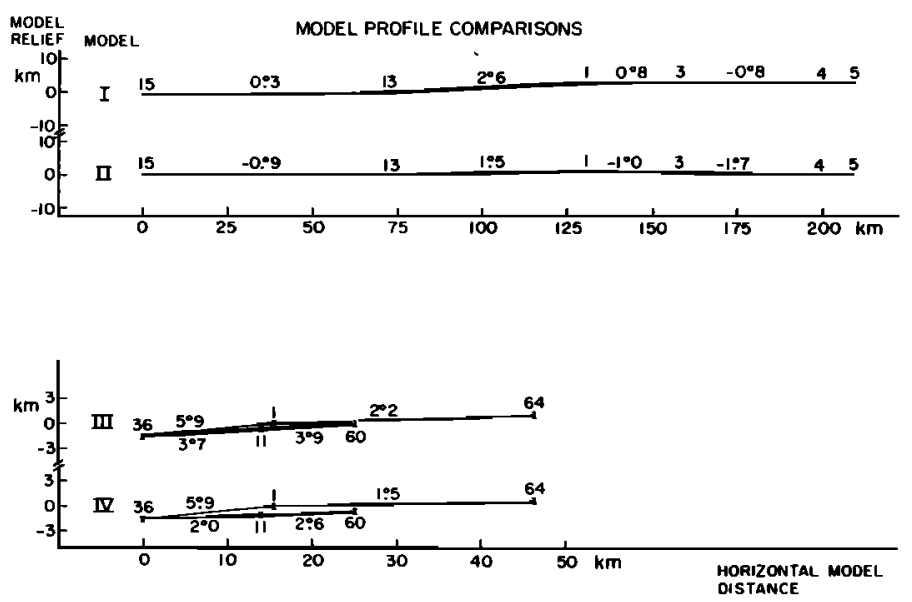

Fig. 4. Vertical profiles for comparison of independent stereo models on Middle Spot (models 1 and 2) and the residual south polar cap (models 3 and 4).

two points. The sample in a model was always too small to calculate a meaningful formal error such as a standard deviation, and so this estimate was adopted. Note that, for most models, this figure is within a factor of 2 of the predicted error $\Delta H$.

A validation of the analytic technique is the essential agreement of independent stereo models except for a general tilt of one model in relation to the other. This is demonstrated to be the case for models 1 and 2 by the profiles in Figure 4. The 15-13 section of each profile is approximately perpendicular to the remainder, and so we are able to estimate the two components of the relative tilt of the models. From the general error for model elevations given in Table 3 we have calculated the slope differences and uncertainties along the profile. These are listed in Table 4. We observe that model 2 disagrees with model 1 only by a relative tilt of about $1.2^{\circ}$ to the west around the $13-5$ axis and $1.2^{\circ}$ to the north about the $13-15$ axis.

Let us now turn our attention to other profiles of Middle Spot in Figure 5. The low

TABLE 4. Systematic Differences in Topography between Models 1 and 2

\begin{tabular}{crrr}
\hline \multirow{2}{*}{$\begin{array}{c}\text { Profile } \\
\text { Section }\end{array}$} & Model 1 & Model 2 & $\begin{array}{c}\text { Slope Difference } \\
\text { Relative Tilt } \\
\text { of Models }\end{array}$ \\
\cline { 2 - 4 } & & & \\
\hline $13-15$ & $0.3^{\circ} \pm 0.2^{\circ}$ & $-0.9^{\circ} \pm 0.3^{\circ}$ & $1.2^{\circ} \pm 0.4^{\circ}$ \\
$13-1$ & $2.6^{\circ} \pm 0.2^{\circ}$ & $1.5^{\circ} \pm 0.3^{\circ}$ & $1.1^{\circ} \pm 0.4^{\circ}$ \\
$1-3$ & $0.8^{\circ} \pm 0.4^{\circ}$ & $-1.0^{\circ} \pm 0.8^{\circ}$ & $1.8^{\circ} \pm 0.9^{\circ}$ \\
$3-4$ & $-0.8^{\circ} \pm 0.3^{\circ}$ & $-1.7^{\circ} \pm 0.5^{\circ}$ & $0.9^{\circ} \pm 0.6^{\circ}$ \\
\hline
\end{tabular}

slopes on the flanks of the cone and the total relief of about $10 \mathrm{~km}$ point to the basaltic shield volcano as the closest earthly analog. A group of basaltic shields, the island of $\mathrm{Ha}$ waii, stands approximately $10 \mathrm{~km}$ above the sea floor, and unmodified slopes range from $2^{\circ}$ to $12^{\circ}$ [Macdonald, 1972]. The central crater of Middle Spot, approximately $47 \mathrm{~km}$ across, is, however, much larger than those associated with earthly shield volcanoes. For example, the summit caldera of Mauna Loa measures only $2.4 \times 4.8 \mathrm{~km}$.

A second pair of overlapping stereo models, on the residual south polar cap, is presented in Figure 6. Figure 4 contains profiles common to these two models. Again we can account for the differences in the profiles with a simple tilt of one model in relation to the other. The axis of the tilt appears to run through points 36 and 1.

The residual south polar cap is an area of surface frost observed to persist through the southern hemisphere summer in 1971-1972. The dark strips of defrosted terrain running through the cap suggest that the underlying materials are the same as those of a very widespread polar geologic unit now designated laminated terrain [Murray et al., 1972]. The name is derived from the narrow parallel bands that seem to follow the contours of the topography. On pictures of the region of Figure 6 , which have been specially processed to bring out de- 
tail in the dark areas, it is possible to see eight bands in the wide defrosted strip to the east and three bands on the narrow strip in the southwest. If these bands represent stratified geologic units, it becomes important to estimate the total relief across a defrosted strip to place some constraints on the depositional process. We will attempt this for the narrow defrosted strip using the profiles for model 4 in Figure 5.

Because surfaces of equal elevation in our topographic model differ from such surfaces on Mars only by a small tilt, we can estimate the relief across the dark strips only if we systematically untilt straight profiles through at least three points. For example, profile 65-69-92 is shown in Figure 5. Points 65 and 69 lie on one side of the strip, and point 92 lies on the other. We assume that points 65 and 69 lie in the same horizontal surface and then project that surface on to point 92, which is calculated to lie 175 meters below that reference level. This assumption of very low relief in the continuously frosted area is justified by systemic changes in slope observed on profiles crossing both defrosted strips. Relief across the narrow strip has been calculated for four profiles (Table 5). The stereo model indicates a slope downward toward the southwest with relief of the order of 200 meters.
Before passing on to another Martian feature, we note the anomalous relationship of the general model error to the calculated $\Delta H$ for the south polar cap models. The observed error is approximately twice the predicted error. This discrepancy may be attributed to the nature of the features used in these models. Instead of fixed point features such as crater centers, which were commonly used elsewhere on Mars, we have used dark spots of defrosted ground and albedo markings within the frost. Such features have the unfortunate property of changing size and shape significantly with time, tending to increase $\Delta H^{\prime}$.

Model $\mathbf{5}$ shown in Figure $\mathbf{7}$ is another Martian volcano, Nix Olympica. The slopes shown in the profiles of Figure 5 are similar in angle to those of Middle Spot, but the base of the cone is larger, and so the total relief from the top of the basal scarp (point 9) to the rim of the complex caldera is about $20 \mathrm{~km}$, twice that of any similar feature on the earth.

Model 6 shown in Figure 8 represents the limits of precision attainable from Mariner 9 data. A pair of high-resolution pictures taken near periapsis were used in its construction. The area is part of the Coprates canyon or trough system that stretches $4800 \mathrm{~km}$ across Mars between the equator and $20^{\circ} \mathrm{S}$. The plateau shown sits inside a trough over $200 \mathrm{~km}$



Fig. 5. Vertical profiles of Martian surface features from six stereo models. 


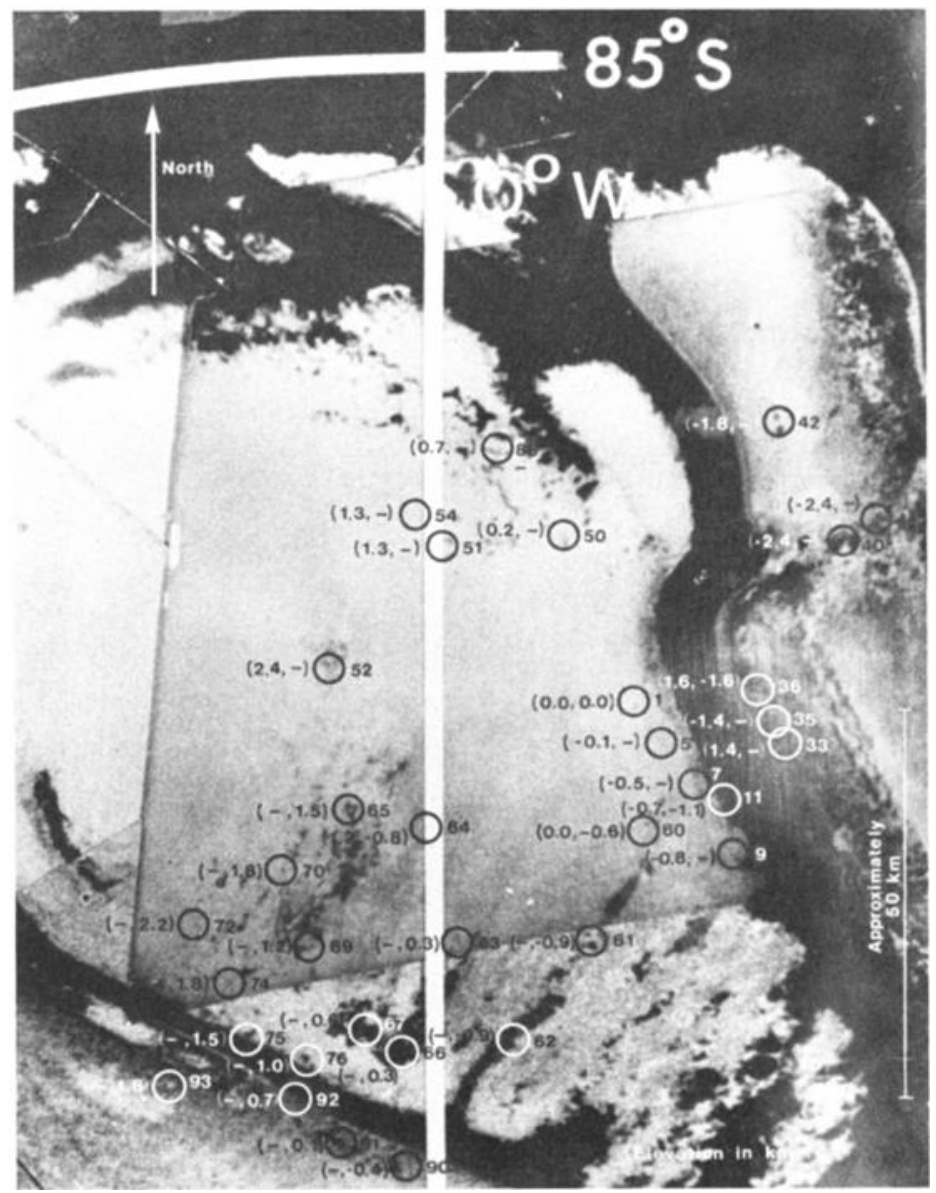

Fig. 6. A mosaic of pictures of the residual south polar cap rectified to a polar stereographic projection. Point elevations in parentheses have been determined by analytic photogrammetry from models 3 and $4(3,4)$. (MTVS 4149-21, DAS 06029803; MTVS 4221-06, DAS 08331829; MTVS 4140-27, DAS 05741963; MTVS 4205-102, DAS 07791983.)

wide. The craters on its upper surface resemble those outside the canyon and suggest that this was once part of a continuous stretch of cratered terrain that has been partially de-

TABLE 5. Estimating Relief across a Defrosted Strip within the Residual South Polar Cap

\begin{tabular}{lcc}
\hline & $\begin{array}{c}\text { Points } \\
\text { Held } \\
\text { Leve1 }\end{array}$ & $\begin{array}{c}\text { Derived Relief } \\
\text { across } \\
\text { Defrosted Strip, } \\
\text { meters }\end{array}$ \\
\hline $61-66-92$ & 61,66 & 270 \\
$67-76-93$ & 67,76 & 54 \\
$65-69-92$ & 65,69 & 175 \\
$64-67-91$ & 64,67 & 400 \\
\hline
\end{tabular}

stroyed by the canyon-forming process. The local relief, about $3.5 \mathrm{~km}$, represents material removed to create the trough. The slopes, up to at least $13.9^{\circ}$, are distinctly steeper than those seen on the flanks of the volcanoes but are not particularly steep by earth standards.

Although it has demonstrated a useful technique and tested the quality of topographic data from Mariner 9 pictures, this paper has made only a small contribution to data reduction. We must now start the task of sorting out the stereo coverage on interesting features and determining which photogrammetric techniques to apply. Such work will certainly take years, and the larger task of geologic interpretation will proceed for decades. 


\section{APPEndIX}

A scheme for describing the geometry of oblique pictures. Figure 9 contains all the quantities that are important for the determination of relief between point features seen in an oblique picture. For this report I shall merely present the relations derived from this diagram and defer an outline of their derivation to another place. The relief shown in Figure 9 is the vertical distance $H$, which is related to other quantities in the diagram by the basic relation

$H=\left(H^{\prime} \cdot S R\right) / x \cos (S U B R) / \sin (V A R)$

where the quantities are defined:

$H$ vertical relief to be determined.

$H^{\prime}$ relief displacement of the crest of $H$ from its foot, i.e., the length of the image of $H$ in the focal plane.

$S R$ slant range, from the camera to the feature $H$. $x$ distance from the optic center of the lens to the image of $H$ in the focal plane.

SUBR angle between a line, perpendicular to the direction of $H^{\prime}$ and passing through the optic center of the lens, and the optic path to feature $H$.

$V A R$ emission angle at the surface at feature $H$.

The quantities $S R, x, S U B R$, and $V A R$ from which $H$ is calculated are all derived from a more fundamental set of quantities describing the position and orientation of the camera when photographing $H$. There is a great deal of flexibility in the choice of this set, but, as an example, we shall use the following:

$R$ radius of the planet at feature $H$.

$R M A G$ range from the spacecraft to the center of the planet.

$T A$ tilt angle, the angle between the camera axis and the direction to the center of the planet.

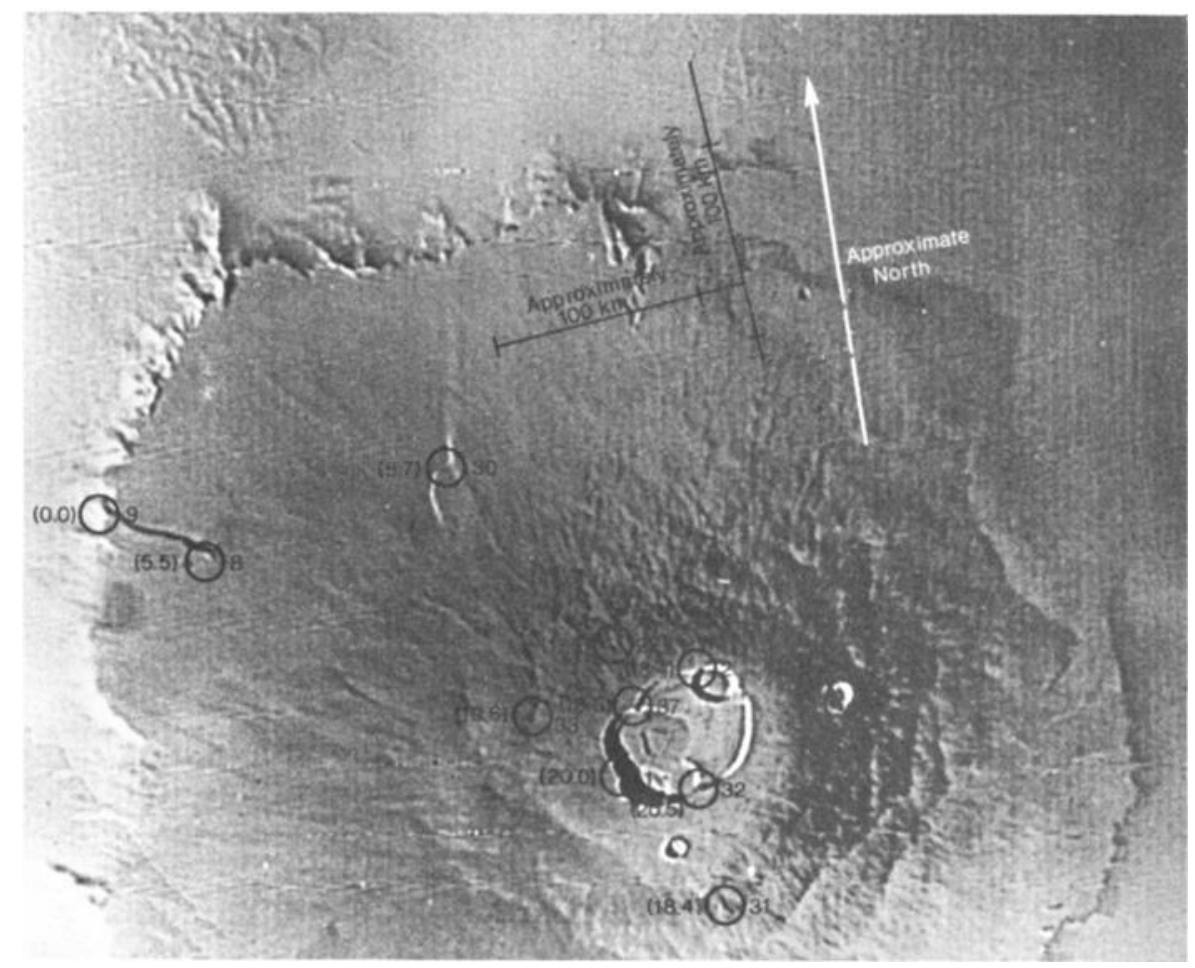

Fig. 7. A slightly oblique view of part of the volcanic shield Nix Olympica. Point elevations in parentheses have been determined by analytic photogrammetry. (MTVS 4174-93, DAS 06823918.) 


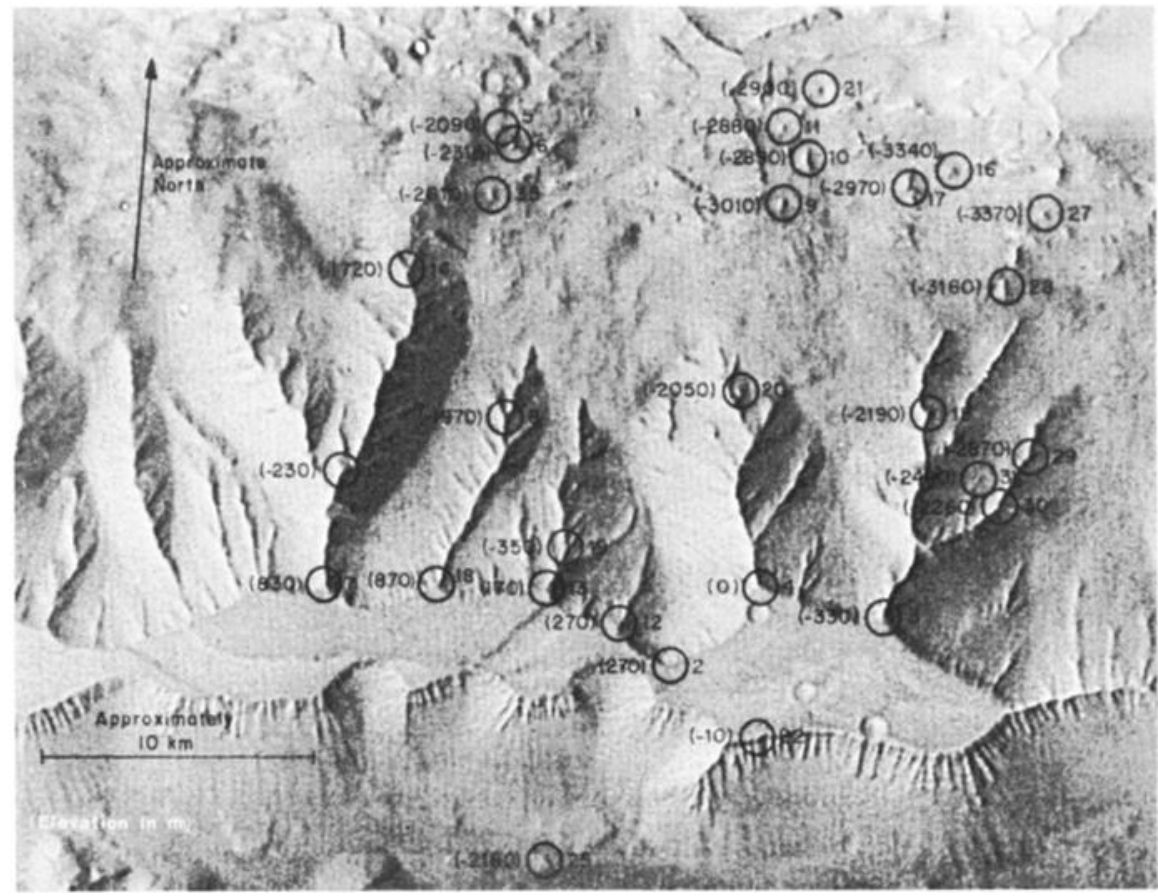

Fig. 8. A nearly vertical view of a flat topped ridge rising from the bottom of the Coprates trough or canyon. Point elevations in parentheses have been determined by analytic photogrammetry. (MTVS 4191-42, DAS 07326763.)

\section{$f$ focal length of the camera.}

$L, K$ coordinates of the image of feature $H$ in the focal plane.

The quantities in (A1) are related to the above by:

$$
\begin{aligned}
& x=\left(K^{2}+L^{2}+f^{2}\right)^{1 / 2} \\
& S U B R=\tan ^{-1}[(S I-S I A) / x] \\
& S I \equiv\left\{K^{2}+[f \cdot \tan (T A)+L]^{2}\right\}^{1 / 2} \\
& S I A \equiv f \cdot \tan (T A)\left[1-(K / S I)^{2}\right]^{1 / 2}(\mathrm{~A} 3 c) \\
& V A R=\sin ^{-1}[(R M A G / R) \cdot \sin (N A A)] \\
& N A A \equiv S U B R+S U A \\
& S U A \equiv \tan ^{-1}(S I A / x) \\
& S R=R \cdot \sin (V A R-N A A) / \sin (N A A)
\end{aligned}
$$

The relationships among the quantities of
(A3), (A4), and (A5) are exhibited in Figures 10 and 11 .

The determination of relief of surface features. Because real geologic features do not consist of upright posts or vertical scarps that would be imaged as $H$ is in Figure 9, we must somehow infer a relief displacement $H^{\prime}$ for a feature, for example, a hilltop, that is the image of the relief of the hilltop above some point at its base. This is possible if we have a second picture of the same feature taken from a different perspective. Figure 12 consists of schematic diagrams of two such pictures of a hypothetical feature. Points $A$ and $B$ represent point features at the crest and foot of a hill, respectively, imaged in two separate pictures. We wish to derive the vertical relief along traverse $A B$. This is imaged as $H_{1}^{\prime}$ in $(a)$ and $H_{2}^{\prime}$ in $(b)$, but the point $C$ defining the length of $H_{1}^{\prime}$ and $H_{2}^{\prime}$ is not imaged in the pictures as it is below the ground surface vertically beneath point $A$ at the elevation of point $B$. The location of point $C$ in a picture is inferred from two facts. (1) It is located on a line joining $A$ and the nadir 




Fig. 9. The geometry of relief $H$ imaged in a planetary scale oblique picture (partly based on Imhof and Doolittle [1966]).

point $N^{\prime}$. An axiom of photogrammetry, which is obvious from Figure 9, is that relief displacement is radially outward from the nadir point. (2) The ground distance $\overline{B C}$ derived from the image lengths $\overline{B C}_{1}^{\prime}$ and $\overline{B C}_{2}^{\prime}$ in the pictures must be the same. The problem now is to find a pair of distances in the focal plane, $H_{1}{ }^{\prime}$, and $H_{2}{ }^{\prime}$, which represent the same value

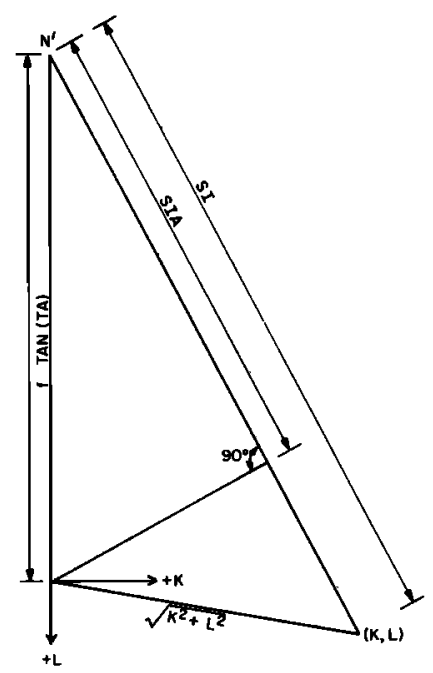

Fig. 10. The geometry of some quantities in the focal plane of an oblique picture. Some relations are given by (A3) and (A4).



Fig. 11. The geometry of some quantities in the principal plane (the plane containing the camera axis and the nadir) of an oblique picture (A5).

of $H$ according to (A1) and give identical horizontal ground distances from the consequent image lengths $\overline{B C}_{1}^{\prime}$ and $\overline{B C}_{2}^{\prime}$. Because of the nonlinear nature of (A1)-(A5), I have adopted an iteration procedure. Briefly it is: (1) pick an $H_{1}{ }^{\prime}$ and calculate $H$ from (A1), (2) calculate $H_{2}^{\prime}$ from (A1), (3) calculate the ground distance $\overline{B C}$ for each photo from $\overline{B C}_{1}^{\prime}$ and $\overline{B C}_{2}^{\prime}$,

(o)

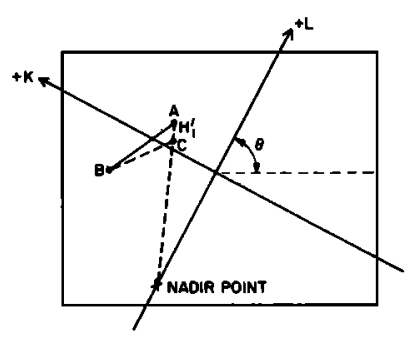

(b)

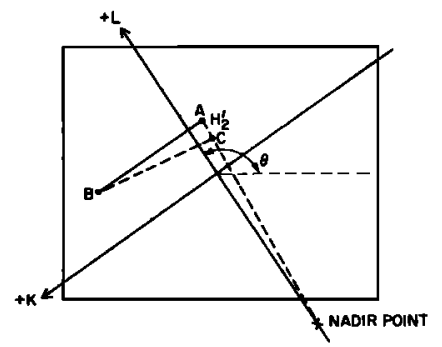

Fig. 12. Schematic diagrams of a topographic feature observed in two oblique pictures. 
and (4) use the value of $\left(\overline{B C}_{1}-\overline{B C}_{2}\right)$ to correct $H_{1}{ }^{\prime}$.

All that remains to implement the above procedure is to outline a technique for the derivation of the horizontal ground distance $\overline{B C}$ from its image (step 3) as above. One scheme is to find the difference of vectors from the spacecraft to $B$ and $C$. The coordinate system of these vectors, centered at 0 , is shown in Figure 9 . If we label the vectors to $B$ and $C$ as $\mathbf{B}$ and $\mathbf{C}$, respectively, their components can be derived from Figure 9 as:

$$
\begin{aligned}
B_{X} & =S R_{B} \cdot \sin \left(N A A_{B}\right) \cdot \sin \left(B T_{B}\right) \\
N A_{B} & \equiv L+f \cdot \tan (T A) \\
B T_{B} & =\tan ^{-1}\left[-K_{B} /\left(N A_{B} \cdot \cos (T A)\right)\right] \\
B_{Y} & =S R_{B} \cdot \sin \left(N A A_{B}\right) \cdot \cos \left(B T_{B}\right) \\
B_{Z} & =S R_{B} \cdot \cos \left(N A A_{B}\right)
\end{aligned}
$$

The components of $\mathbf{C}$ are similarly derived with the appropriate $C$ subscript on parameters. The cord distance from $B$ to $C$ is just $|\mathbf{B}-\mathbf{C}|$. This deviates from the exact distance $\overline{B C}$, following the curvature of the planet, by less than 1 part in $10^{3}$ for $\overline{B C} \lesssim 350 \mathrm{~km}$ on Mars. This is adequate for out work on generally small features.

Consistency arguments to improve input parameters. If the values of the basic input parameters describing the geometry of a picture, $R M A G, T A$, and the orientation angle $\theta$ of the $K-L$ coordinate system in Figure 12, are sufficiently imprecise, the elevations derived from point features may be contradictory. Let us designate as $H(a, b)$ the derived elevation of point $a$ above point $b$. The contradictions we find are of the form of

$$
H(1,2)-H(1,3) \neq H(4,2)-H(4,3)
$$

Given a sufficient number of point features in the stereo overlap region of a pair of pictures, we can devise any number of conditions, such as

$$
H(1,2)-H(1,3)=H(4,2)-H(4,3)
$$

to control an iteration scheme to improve the values of any or all of $R M A G, T A$, and $\theta$. The procedure is to hold these quantities constant for one of the pictures and let them vary for the other until conditions such as (A8) are satisfied to the desired accuracy. This procedure is commonly termed a relative orienta- tion. It was required to establish models 3,4 , and 5 presented in the main body of this paper. Changes required in $\theta$ and $T A$ were less than $0.5^{\circ}$ in all cases, and only one model required adjustment in the nominal value of $R M A G$. Because the parameters of one picture were held constant, the resulting three-dimensional model of topography may have an overall tilt in relation to the local horizontal reflecting errors in $T A$ and $\theta$ for that picture.

Caution must be exercised in the performance of a relative orientation with extremely narrow angle photography, such as that from the Mariner 9 narrow angle camera. D. W. G. Arthur (personal communication, 1973) pointed out that, in the extreme case, imaginary orthographic pictures, the angle between the camera stations measured from the ground target is indeterminate. The magnitude of this angle controls the scale of the stereo model, and so it should be held constant while performing a relative orientation. This constraint has been maintained in the construction of models in this paper that were derived from narrow angle photography.

Arthur has shown that the above restriction can be avoided if a minimum of three pictures of a feature can be employed. This author has not pursued this possibility, owing to the extreme rarity of such triple coverage by the Mariner 9 narrow angle camera.

Acknowledgments. I am indebted to all the people who combined their efforts to make Mariner Mars 1971 a successful mission. With respect to the work reported in this article, specific acknowledgment for aid must go to L. A. Soderblom and S. S. C. Wu of the U.S. Geological Survey, M. Benesh of the Jet Propulsion Laboratory, and B. Murray of the California Institute of Technology. The processed pictures used for this work were obtained and studied in a timely fashion only with the cooperation of J. Seidman, A. Schwartz, and J. Soha of JPL and J. J. van der Woude of Caltech.

\section{ReFERENCES}

Imhof, R. K., and R. C. Doolittle, Mapping from oblique photographs, in Manual of Photogrammetry, p. 913, American Society of Photogrammetry, Falls Church, Va., 1966.

Macdonald, G. A., Volcanoes, Prentice-Hall, New Jersey, 510 pp., 1972.

Masursky, H., R. Batson, W. Borgeson, M. Carr, J. McCauley, D. Milton, R. Wildey, D. Wilhelms, B. Murray, N. Horowitz, R. Leighton, 
R. Sharp, W. Thompson, G. Briggs, P. Chandeysson, E. Shipley, C. Sagan, J. Pollack, J. Lederberg, E. Levinthal, W. Hartmann, T. McCord, B. Smith, M. Davies, G. deVaucouleurs, and C. Leovy, Television experiment for Mariner Mars 1971, Icarus, 12, 10, 1970.

Murray, B. C., L. A. Soderblom, J. A. Cutts, R. P. Sharp, D. J. Milton, and R. B. Leighton, Geological framework of the south polar region of Mars, Icarus, 17, 328, 1972.
Snyder, L. M., Mariner 9 TV subsystem calibration report, Doc. 610-202, Jet Propul. Lab., Pasadena, Calif., 1971.

Wu, S. S. C., F. J. Schafer, G. M. Nakata, R. Jordan, and $\mathbf{K}$. R. Blasius, Photogrammetric evaluation of Mariner 9 photography, J. Geophys. Res., 78, this issue, 1973.

(Received January 4, 1973; revised March 14, 1973.) 